PROCEEDINGS OF THE

AMERICAN MATHEMATICAL SOCIETY

Volume 124, Number 12, December 1996, Pages 3711-3719

S 0002-9939(96)03446-6

\title{
EXPANDING THE JOINT SPECTRUM OF PAIRS OF COMMUTING CONTRACTIONS
}

\author{
ADRIAN IONESCU
}

(Communicated by Theodore W. Gamelin)

\begin{abstract}
We present a connection between solving the invariant subspace problem for a single operator $T$ on Hilbert space and the existence of a common invariant subspace for two commuting related operators. In particular, we reduce the problem of the existence of nontrivial invariant subspaces for a single contraction with spectral radius one to the problem of the existence of common nontrivial invariant subspaces for a pair of commuting contractions with large joint spectra.
\end{abstract}

\section{INTRODUCTION}

During the past fifteen years important progress has been made on the problem of finding nontrivial invariant subspaces for a contraction of spectral radius one acting on a Hilbert space. In particular, it was shown in [5] that every contraction whose spectrum contains the unit circle has a nontrivial invariant subspace. The problem remains open if the spectrum touches the unit circle. Recently, interesting results have been obtained concerning the problem of the existence of nontrivial common invariant subspaces for pairs (or $k$-tuples) of commuting operators [1], [6], [16]. Thus it becomes natural to ask what impact the problem of finding nontrivial common invariant subspaces for pairs (or $k$-tuples) of commuting contractions may have on the problem of finding nontrivial invariant subspaces for a single contraction of spectral radius one. This note presents a modest contribution in this direction.

\section{Preliminaries}

Let $\mathbb{D}$ denote the open disc in the complex plane $\mathbb{C}$ and $\mathbb{T}=\partial \mathbb{D}$ the unit circle. Let $\mathbb{N}$ be the set of positive integers and $\mathbb{R}$ the set of real numbers. We write $\mathbf{H}^{\infty}\left(\mathbb{D}^{2}\right)$ as the algebra of bounded analytic functions on $\mathbb{D}^{2}$. As usual, we say that a subset $\Delta$ in $\mathbb{D}$ is dominating for an arc $E$ in $\mathbb{T}$ if almost every point of $E$ is a nontangential limit of a sequence of points from $\Delta$. It is known that $\Delta$ in $\mathbb{D}$ is dominating for $\mathbb{T}$ if and only if $\|u\|_{\infty}=\sup _{z \in \Delta}|u(z)|$, for all $u \in \mathbf{H}^{\infty}(\mathbb{D})$ [7]. Similarly, we say that $\Delta$ in $\mathbb{D}^{2}$ is dominating for $\mathbb{T}^{2}$ if and only if $\|u\|_{\infty}=\sup _{z \in \Delta}|u(z)|$, for all $u \in \mathbf{H}^{\infty}\left(\mathbb{D}^{2}\right)$. Let $\mathcal{L}(\mathcal{H})$ be the algebra of bounded linear operators on a separable, complex infinite dimensional Hilbert space $\mathcal{H}$. We say that a contraction operator $T$ is a (BCP)-operator if $\sigma_{e}(T)$ is dominating on $\mathbb{T}$ (see [4]). In [17], Ch.III, Sz.-Nagy and Foias developed an $\mathbf{H}^{\infty}(\mathbb{D})$ functional calculus for any absolutely continuous

Received by the editors August 24, 1994 and, in revised form, May 9, 1995.

1991 Mathematics Subject Classification. Primary 47A15; Secondary 30C20, 30D40, 47A13.

(C)1996 American Mathematical Society 
contraction $T$ in $\mathcal{L}(\mathcal{H})$ (notation: (ACC)). A two-variable version of the Nagy-Foiaş functional calculus was constructed in [3].

Let $\mathcal{L}(\mathcal{H})_{\text {comm }}^{(2)}$ be the set of commuting pairs $\left(T_{1}, T_{2}\right)$. We define $\sigma_{l e}\left(T_{1}, T_{2}\right)$, the joint left essential spectrum, to be the set of all points $\left(\lambda_{1}, \lambda_{2}\right)$ in $\mathbb{C}^{2}$ for which there exists an orthonormal sequence $\left\{x_{n}\right\} \subset \mathcal{H}$ such that for $j=1,2,\left\|\left(T_{j}-\lambda_{j}\right) x_{n}\right\| \stackrel{n}{\rightarrow}$ 0 . For the definition of the joint right essential spectrum, joint Harte spectrum (notation: $\sigma_{H}\left(T_{1}, T_{2}\right)$ ), joint Taylor spectrum (notation: $\sigma_{T}\left(T_{1}, T_{2}\right)$ ) and other results regarding the Harte spectrum and Taylor spectrum we refer to [10].

If $\mathcal{M}$ is a subspace of $\mathcal{H}$ and $T=\left(T_{1}, T_{2}\right)$ in $\mathcal{L}(\mathcal{H})_{\text {comm }}^{(2)}$, then $\mathcal{M}$ is called a common invariant subspace for $\left(T_{1}, T_{2}\right)$ if $T_{j} \mathcal{M} \subseteq \mathcal{M}$, for $j=1,2$. The lattice of all common invariant subspaces of $T$ will be denoted by $\operatorname{Lat}\left(T_{1}, T_{2}\right)$. The lattice of all hyperinvariant subspaces of $\left(T_{1}, T_{2}\right)$ (i.e., the set of all subspaces $\mathcal{M}$ invariant for all operators that commute with $T_{1}$ and $\left.T_{2}\right)$ will be denoted by $\operatorname{HLat}\left(T_{1}, T_{2}\right)$.

In the one-variable case, by various conformal mappings on snake-like Carathéodory domains, it is proved in [13], [14] that for every subarc $E$ of $\mathbb{T}$, and for every completely nonunitary contraction $T$ with connected spectrum and spectral radius one there exists a contraction $T^{\prime}$ such that

- $\sigma_{l e}\left(T^{\prime}\right) \cap \mathbb{T} \supseteq E$

- $\sigma_{l e}\left(T^{\prime}\right)$ is dominating for the arc $E$;

- $\operatorname{Lat}(T)=\operatorname{Lat}\left(T^{\prime}\right)$.

Then, it results that there exists a bounded analytic function $f$ on $\mathbb{D}^{-}$such that for every contraction $T$ with $1 \in \sigma(T)$ we have $\sigma[f(T)]=\mathbb{D}^{-}$. Therefore, if it is true that every completely nonunitary contraction in $\mathcal{L}(\mathcal{H})$ whose spectrum is the closed unit disc has a nontrivial hyperinvariant subspace, then every nonscalar contraction in $\mathcal{L}(\mathcal{H})$ with spectral radius one has a nontrivial hyperinvariant subspace. We also present variants of the above results in two variables (however, most of the results presented in the rest of the paper can be easily stated for more than two variables).

\section{Joint SPECTRA AND SPECTRAL PROPERTIES}

The results in this section present short proofs of some spectral mapping properties for the joint spectrum of pairs of commuting contractions (see also [11], [20]). These results will be used in the constructions made in Section 4 .

Proposition 3.1. Let $T_{1}$ and $T_{2}$ be commuting completely nonunitary contractions and let $u_{1}, u_{2} \in \mathbf{H}^{\infty}\left(\mathbb{D}^{2}\right)$. For any $\left(\zeta_{1}, \zeta_{2}\right) \in \sigma_{H}\left(T_{1}, T_{2}\right) \cap \mathbb{D}^{2}$,

$$
\left(u_{1}\left(\zeta_{1}, \zeta_{2}\right), u_{2}\left(\zeta_{1}, \zeta_{2}\right)\right) \in \sigma_{H}\left(u_{1}\left(T_{1}, T_{2}\right), u_{2}\left(T_{1}, T_{2}\right)\right),
$$

and a corresponding statement is valid for the joint left essential spectrum of $T_{1}$ and $T_{2}$.

Proof. We show that (3.1) is valid for the joint Harte spectrum; a similar argument works for the joint left essential spectrum. Let $\left(\zeta_{1}, \zeta_{2}\right) \in \sigma_{H}\left(T_{1}, T_{2}\right) \cap \mathbb{D}^{2}$, and suppose, by way of contradiction, that we have

$$
\left(u_{1}\left(\zeta_{1}, \zeta_{2}\right), u_{2}\left(\zeta_{1}, \zeta_{2}\right)\right) \notin \sigma_{H}\left(u_{1}\left(T_{1}, T_{2}\right), u_{2}\left(T_{1}, T_{2}\right)\right) .
$$

Then the pair $\left(u_{1}\left(T_{1}, T_{2}\right)-u_{1}\left(\zeta_{1}, \zeta_{2}\right), u_{2}\left(T_{1}, T_{2}\right)-u_{2}\left(\zeta_{1}, \zeta_{2}\right)\right)$ is invertible, i.e., there exist operators $A_{1}, A_{2} \in \mathcal{L}(\mathcal{H})$ such that

$$
I_{\mathcal{H}}=A_{1}\left[u_{1}\left(T_{1}, T_{2}\right)-u_{1}\left(\zeta_{1}, \zeta_{2}\right)\right]+A_{2}\left[u_{2}\left(T_{1}, T_{2}\right)-u_{2}\left(\zeta_{1}, \zeta_{2}\right)\right],
$$


and similarly for the right invertibility. Moreover, using the Gleason factorization (cf., [19], p.114) for the class $\mathbf{H}^{\infty}\left(\mathbb{D}^{2}\right)$ and the functional calculus defined in [3] we have

$$
u_{j}\left(T_{1}, T_{2}\right)-u_{j}\left(\zeta_{1}, \zeta_{2}\right)=g_{j}\left(T_{1}, T_{2}\right)\left(T_{1}-\zeta_{1}\right)+h_{j}\left(T_{1}, T_{2}\right)\left(T_{2}-\zeta_{2}\right),
$$

where for each $j=1,2, g_{j}$ and $h_{j}$ are in $\mathbf{H}^{\infty}\left(\mathbb{D}^{2}\right)$. Therefore, by (3.2) and (3.3), we obtain the left invertibility of the pair $\left(T_{1}, T_{2}\right)$

$$
\begin{aligned}
I_{\mathcal{H}}= & {\left[A_{1} g_{1}\left(T_{1}, T_{2}\right)+A_{2} g_{2}\left(T_{1}, T_{2}\right)\right]\left(T_{1}-\zeta_{1}\right) } \\
& +\left[A_{1} h_{1}\left(T_{1}, T_{2}\right)+A_{2} h_{2}\left(T_{1}, T_{2}\right)\right]\left(T_{2}-\zeta_{2}\right),
\end{aligned}
$$

and similarly we prove the right invertibility of $\left(T_{1}, T_{2}\right)$. Thus, $\left(\zeta_{1}, \zeta_{2}\right) \notin \sigma_{H}\left(T_{1}, T_{2}\right)$ and this is a contradiction.

Corollary $3.2([11])$. Let $T$ be a completely nonunitary contraction in $\mathcal{L}(\mathcal{H})$. Then, for any $\left(u_{1}, u_{2}\right)$ in $\mathbf{H}^{\infty}(\mathbb{D})$ we have that for any $z \in \sigma_{H}(T) \cap \mathbb{D}$,

$$
\left(u_{1}(z), u_{2}(z)\right) \in \sigma_{H}\left(u_{1}(T), u_{2}(T)\right),
$$

and a corresponding statement is valid for the joint left essential spectrum of $T$.

The following is a known result (cf., [15], p.225):

Lemma 3.3. Let $u$ in $\mathbf{H}^{\infty}(\mathbb{D})$ be such that $u$ has a continuous extension to $e^{i t_{0}}$ (which we continue to denote by $u$ ). Then, there exists a sequence of functions $\left\{u^{(n)}\right\} \subset \mathbf{H}^{\infty}(\mathbb{D})$ with $\left\|u-u^{(n)}\right\|_{\infty} \stackrel{n}{\rightarrow} 0$, and each $u^{(n)}$ is analytic on a neighborhood of $z=e^{i t_{0}}$.

We also remark that one can prove a two-dimensional variant of Lemma 3.3. The proof follows the ideas used in [2], [15], Ch.VIII; therefore, we only state the theorem.

Theorem 3.4. If $u \in \mathbf{H}^{\infty}\left(\mathbb{D}^{2}\right)$ and $u$ is continuously extendible to a point $w=$ $\left(w_{1}, w_{2}\right)$ in $\mathbb{T}^{2}$, then there exists a sequence of analytic functions $\left\{u^{(n)}\right\}$ in $\mathbf{H}^{\infty}\left(\mathbb{D}^{2}\right)$ with $\left\|u-u^{(n)}\right\|_{\infty} \stackrel{n}{\rightarrow} 0$ such that each $u^{(n)}$ is analytic on a neighborhood of $w$.

Note that Theorem 3.4 is no longer true if we consider a point in $\partial \mathbb{D}^{2} \backslash \mathbb{T}^{2}$. In the proof of the next spectral-mapping theorem we will use only Lemma 3.3.

Theorem 3.5. Let $\left(T_{1}, T_{2}\right)$ be a pair of commuting operators in $(A C C)$ and let $\left(w_{1}, w_{2}\right) \in \sigma_{H}\left(T_{1}, T_{2}\right) \cap \mathbb{T}^{2}$. Let $u=\left(u_{1}, u_{2}\right)$ be a pair of functions in $\mathbf{H}^{\infty}(\mathbb{D})$ such that for each $j=1,2, u_{j}$ has a continuous extension to $\mathbb{D} \cup\left\{w_{j}\right\}$ (which we continue to denote by $\left.u_{j}\right)$. Then

$$
\left(u_{1}\left(w_{1}\right), u_{2}\left(w_{2}\right)\right) \in \sigma_{H}\left(u_{1}\left(T_{1}\right), u_{2}\left(T_{2}\right)\right),
$$

and a similar statement holds for the joint left essential spectrum.

Proof. Suppose by way of contradiction that $\left(u_{1}\left(w_{1}\right), u_{2}\left(w_{2}\right)\right)$ does not belong to the joint Harte spectrum of the pair $\left(u_{1}\left(T_{1}\right), u_{2}\left(T_{2}\right)\right)$. Then, there exist operators $A_{1}, A_{2} \in \mathcal{L}(\mathcal{H})$ such that

$$
I_{\mathcal{H}}=A_{1}\left[u_{1}\left(T_{1}\right)-u_{1}\left(w_{1}\right)\right]+A_{2}\left[u_{2}\left(T_{2}\right)-u_{2}\left(w_{2}\right)\right]
$$

and similarly for the right invertibility. Using Lemma 3.3 for each $j=1,2$, we may choose sequences $\left\{u_{j}^{(n)}\right\}_{n}$ in $\mathbf{H}^{\infty}(\mathbb{D})$ such that each $u_{j}^{(n)}$ is analytic in a neighborhood of $w_{j}$ and $\left\|u_{j}-u_{j}^{(n)}\right\|_{\infty} \stackrel{n}{\rightarrow} 0$. Then, by the existence of an $\mathbf{H}^{\infty}(\mathbb{D})$-functional 
calculus for (ACC) (cf., [17], p.117), we obtain that for $j=1,2$,

$$
u_{j}^{(n)}\left(T_{j}\right)-u_{j}^{(n)}\left(w_{j}\right)=\left(T_{j}-w_{j}\right) g_{j}^{(n)}\left(T_{j}\right),
$$

where each $g_{j}^{(n)}$ belongs to $\mathbf{H}^{\infty}(\mathbb{D})$ and is analytic in a neighborhood of $w_{j}$. For $n \in \mathbb{N}$ sufficiently large these factorizations yield

$$
I_{\mathcal{H}}=A_{1}^{(n)} g_{1}^{(n)}\left(T_{1}\right)\left(T_{1}-w_{1}\right)+A_{2}^{(n)} g_{2}^{(n)}\left(T_{2}\right)\left(T_{2}-w_{2}\right),
$$

where $A_{1}^{(n)}, A_{2}^{(n)} \in \mathcal{L}(\mathcal{H})$. Similarly we prove right invertibility, and so we get that $\left(w_{1}, w_{2}\right) \notin \sigma_{H}\left(T_{1}, T_{2}\right)$, which is a contradiction.

Let us prove the result for the joint left essential spectrum. Let $\left\{x_{n}\right\}$ be an orthonormal sequence in $\mathcal{H}$ such that, for each $j=1,2,\left\|\left(T_{j}-w_{j}\right) x_{n}\right\| \stackrel{n}{\rightarrow} 0$. For any $\epsilon>0$ there exists $L=L_{\epsilon}$ in $\mathbb{N}$ such that, for $j=1,2,\left\|u_{j}-u_{j}^{(L)}\right\|_{\infty}<\frac{\epsilon}{3}$. Moreover, there exists $N=N_{\epsilon} \in \mathbb{N}$ such that for $n>N$ we have $\left\|\left(T_{j}-w_{j}\right) x_{n}\right\|<\frac{\epsilon}{3\left\|g_{j}^{(L)}\right\|_{\infty}}$, where $g_{j}^{(L)}$ is given by (3.4). Then for $n>N$ we get that

$$
\begin{aligned}
& \left\|\left[u\left(T_{j}\right)-u\left(w_{j}\right)\right] x_{n}\right\| \leq\left\|\left[u\left(T_{j}\right)-u_{j}^{(L)}\left(T_{j}\right)\right] x_{n}\right\| \\
+ & \left\|\left[u\left(w_{j}\right)-u_{j}^{(L)}\left(w_{j}\right)\right] x_{n}\right\|+\left\|\left[u_{j}^{(L)}\left(T_{j}\right)-u_{j}^{(L)}\left(w_{j}\right)\right] x_{n}\right\| \\
< & \left\|\left(u-u_{j}^{(L)}\right)\left(T_{j}\right) x_{n}\right\|+\frac{\epsilon}{3}+\left\|g_{j}^{(L)}(T)\right\|\left\|\left(T_{j}-w_{j}\right) x_{n}\right\| \\
< & \left\|u-u_{j}^{(L)}\right\|_{\infty}+\frac{\epsilon}{3}+\left\|g_{j}^{(L)}\right\|_{\infty} \frac{\epsilon}{3\left\|g_{j}^{(L)}\right\|_{\infty}}<\epsilon,
\end{aligned}
$$

and the result follows.

Remark 3.6. Let us note the following:

(1) In [11] a similar result is proved for $T_{1}=T_{2}=T$ where $T$ is a completely nonunitary contraction. See also [20], Theorem 2.6(ii).

(2) For $k=1$, we obtain a result that appears in [12] and was a key argument in proving the main results in [13] and [14].

(3) By a result of Cho-Takeguchi, relating the boundaries of the Harte spectrum and the Taylor spectrum (see [10], Theorem 6.6), the previous result is valid if one replaces the joint Harte spectrum with the joint Taylor spectrum.

\section{The JOINT SPECTRUM AND INVARIANT SUBSPACES}

Recall that the cluster set of a complex valued function $u$ in $\Omega$ at $z_{0} \in \Omega^{-}$is the set $([9]$, p.1)

$$
\mathcal{C}\left(u, z_{0}\right)=\left\{w \in \mathbb{C}: \exists\left\{\lambda_{n}\right\}_{n \in \mathbb{N}} \subset \Omega, \lambda_{n} \stackrel{n}{\rightarrow} z_{0}, \text { and } u\left(\lambda_{n}\right) \stackrel{n}{\rightarrow} w\right\} .
$$

For a subset $M$ in $\Omega$ and $z_{0} \in M^{-}$we write ([9], p.2)

$$
\mathcal{C}_{M}\left(u, z_{0}\right)=\left\{w \in \mathbb{C}: \exists\left\{\lambda_{n}\right\}_{n \in \mathbb{N}} \subset M, \lambda_{n} \stackrel{n}{\rightarrow} z_{0}, \text { and } u\left(\lambda_{n}\right) \stackrel{n}{\rightarrow} w\right\} .
$$

The following lemma is a known result (cf., [9], p.24, [18]):

Lemma 4.1. There is a function $h$ in $\mathbf{H}^{\infty}(\mathbb{D})$ such that

$$
\mathcal{C}_{\lambda_{r, s}}\left(h, w_{r, s}\right) \supseteq \mathbb{T} \text {, }
$$

where $w_{r, s}=e^{i \frac{r}{2^{s} \pi}}, s \in \mathbb{N}, 0 \leq r \leq 2^{s+1}$, and $\lambda_{r, s}$ is a radial path with an endpoint at $w_{r, s}$. 
In fact, for all pairs $(r, s), s \in \mathbb{N}, 0 \leq r \leq 2^{s+1}, h\left(\lambda_{r, s}\right)$ will be an infinite long spiral curve $\sigma_{r, s}$ accumulating at $\mathbb{T}$. The next lemma is the main technical ingredient used in what follows.

Lemma 4.2. For every subarc $E=E_{\epsilon}=\left\{e^{i t}:-\frac{\epsilon}{2} \leq t \leq \frac{\epsilon}{2}\right\}$ of $\mathbb{T}, 0<\epsilon<2 \pi$, there exists a Carathéodory domain $G \subset \mathbb{C}$, such that $\partial G$ is dominating for $E$. Moreover, for all pairs $(r, s), s \in \mathbb{N}, 0 \leq r \leq 2^{s+1}$, satisfying $w_{r, s} \in E$, there exists an almost complete radial path $\lambda_{r, s}^{0}$ in $\partial G$ with an endpoint at $w_{r, s}$. (By an almost complete radial path we mean a radial path $\lambda_{r, s}$, with the exception of a countable number of line segments of total length less than a given $\epsilon_{r, s}$. )

Proof. We may assume without loss of generality that $E=\mathbb{S}$, where $\mathbb{S}$ is the semicircle of $\mathbb{T}$ centered at 1 . We now define a snake-like Carathéodory domain "converging" radially to $\mathbb{S}$ which has the properties used in [13] and [14], and has some additional characteristics to provide the desired joint spectrum. In fact, this domain looks like a simply connected infinitely long, outward going spiral, having no point in common with the sector of disc $S_{\left[\frac{3 \pi}{4}, \frac{5 \pi}{4}\right]}$ defined by the $\operatorname{arc}\left[e^{i \frac{3 \pi}{4}}, e^{i \frac{5 \pi}{4}}\right] \subset \mathbb{T}$, and having a lot of additional "radial bands" accumulating at $\mathbb{S}$. To this end, for $n \in \mathbb{N}$ let us denote $r_{n}=1-\frac{1}{n}$ and $\delta_{n}=r_{n+1}-r_{n}$, and consider the following closed domains:

$$
\begin{aligned}
L_{n} & =\left\{z \in \mathbb{D}: z=r e^{i t}, r_{2 n-1} \leq r \leq r_{2 n},-\frac{3 \pi}{4} \leq t \leq \frac{\pi}{2}\right\} \\
R_{n} & =\left\{z \in \mathbb{D}: z=r e^{i t}, r_{2 n+1} \leq r \leq r_{2 n+2},-\frac{\pi}{2} \leq t \leq \frac{3 \pi}{4}\right\} \\
L & =\bigcup_{n=1}^{\infty} L_{n}, \\
R & =\bigcup_{n=1}^{\infty} R_{n} .
\end{aligned}
$$

Then, the domain defined by

$$
G_{L, R}=\mathbb{D} \backslash\left(L \cup R \cup S_{\left[\frac{3 \pi}{4}, \frac{5 \pi}{4}\right]}\right)
$$

is a Carathéodory domain. In what follows, we add and delete from $G_{L, R}$ some "tiny bands" defined by

$$
\begin{aligned}
\Delta_{p, n}= & \left\{z \in \mathbb{D}: z=r e^{i t}, \quad r_{2 n-1} \leq r<r_{2 n}-\delta_{p, 2 n}\right. \\
& \left.-\frac{\pi}{2}+\frac{2 p-1}{2^{n}} \pi<t<-\frac{\pi}{2}+\frac{2 p}{2^{n}} \pi, \quad 0 \leq p \leq 2^{n-1}\right\}, \\
\Lambda_{p, n}= & \left\{z \in \mathbb{D}: z=r e^{i t}, \quad r_{2 n} \leq r \leq r_{2 n+1}-\delta_{p, 2 n+1},\right. \\
& \left.-\frac{\pi}{2}+\frac{2 p}{2^{n}} \pi \leq t \leq-\frac{\pi}{2}+\frac{2 p+1}{2^{n}} \pi, \quad 0 \leq p \leq 2^{n-1}\right\} .
\end{aligned}
$$

The positive numbers $\delta_{p, n}$ are to be fixed at the end of the proof. We write $\Delta_{n}=$ $\bigcup_{p=0}^{2^{n-1}} \Delta_{p, n}, \Delta=\bigcup_{n=1}^{\infty} \Delta_{n}$, and similarly for $\Lambda_{n}$ and $\Lambda$, and we define

$$
G=\left[\mathbb{D} \backslash\left(L \cup R \cup S_{\left[\frac{3 \pi}{4}, \frac{5 \pi}{4}\right]} \cup \Lambda\right)\right] \cup \Delta=\left(G_{L, R} \backslash \Lambda\right) \cup \Delta .
$$


Then $G$ is obviously a Carathéodory domain because, for instance, $G_{L, R}$ defined in (4.1) is a Carathéodory domain, and adding $\Delta$ and deleting $\Lambda$ we do not change any of the required boundary properties of $G$ (in fact, on each $L_{n}$ and $R_{n}$, we have only a finite number of bands, and then it follows that the exterior boundary of $G$ is the same as the interior one). Moreover, adding $\Delta$ and deleting $\Lambda$, we have created an almost complete radial path $\lambda_{r, s}^{0}$ in $\partial G$ with an endpoint at each $w_{r, s} \in \mathbb{S}$, where $s \in \mathbb{N}, 0 \leq r \leq 2^{s+1}$. Indeed, by the choice of $\Lambda$ and $\Delta$, their radial boundaries complete themselves in such a way that for each $w_{r, s} \in \mathbb{S}$, there exists an almost radial path $\lambda_{r, s}^{0}$ determined by boundary points in $\Lambda$ and $\Delta$ and having $w_{r, s}$ as one of its end points. Thus, $\partial G$ contains the same almost radial path $\lambda_{r, s}^{0}$, having $w_{r, s}$ as one of its end points.

The only remaining thing to be fixed is the value of each $\delta_{p, n}$. For simplicity we may assume that $\left\{w_{q}\right\}_{q \in \mathbb{N}}$ is an enumeration of the set $\left\{w_{r, s}\right\}_{s \in \mathbb{N}, 0 \leq r \leq 2^{s+1}} \cap \mathbb{S}$. For a fixed $w_{q}$ and $\epsilon=\epsilon_{q}>0$ we may assume that

$$
\lambda_{q}^{0}=\lambda_{q} \backslash \bigcup_{m \geq 1}\left[\left(r_{m}-\delta_{m, q}\right) e^{i \theta_{q}}, r_{m} e^{i \theta_{q}}\right]
$$

where the sequence $\left\{\delta_{m, q}\right\}_{m}$ denotes the corresponding $\delta$ for the sequence of corner points situated on the given almost complete radial path $\lambda_{q}^{0}$, ordered from the center of $\mathbb{D}$ to the boundary point $w_{q}$. By Lemma 4.1 we know that the path $\lambda_{q}$ is mapped onto the spiral curve $\sigma_{q}$. Using the continuity of $h$ on $\lambda_{q}^{0}$ for each $q \in \mathbb{N}$, we may choose $\delta_{m, q}<\frac{\epsilon_{q}}{2^{m}}\left(\sum_{m \geq 1} \delta_{m, q}<\epsilon_{q}\right)$ in such a way that the corresponding endpoints of the curve defined by $h\left(\left[\left(r_{m}-\delta_{m, q}\right) e^{i \theta_{q}}, r_{m} e^{i \theta_{q}}\right]\right)$ have distance between them less than a given $\eta=\eta_{m, q}$.

Theorem 4.3. For every subarc $E=E_{\epsilon}=\left\{e^{i t}:-\frac{\epsilon}{2} \leq t \leq \frac{\epsilon}{2}\right\}$ of $\mathbb{T}$, where $0<$ $\epsilon<2 \pi$, and for any sequence $\left\{w_{n}\right\}$ dense in $E$, there exist functions $u_{1}$ and $u_{2}$ in $\mathbf{H}^{\infty}(\mathbb{D})$ such that for every completely nonunitary contraction $T$ with $1 \in \sigma(T)$,

(1) $\sigma_{l e}\left(u_{1}(T), u_{2}(T)\right) \cap \mathbb{T}^{2} \supseteq E \times \mathbb{T}$;

(2) $\sigma_{\text {le }}\left(u_{1}(T)\right) \cap \mathbb{D}$ is dominating on $E, \sigma_{l e}\left(u_{2}(T)\right) \cap \mathbb{D}$ is dominating on $\mathbb{T}$, and, moreover, for each $n \in \mathbb{N}$ we have that $\pi_{2}\left(R_{w_{n}} \cap \sigma_{l e}\left(u_{1}(T), u_{2}(T)\right)\right)$ is dominating on $\mathbb{T}\left(\pi_{2}\right.$ is the projection on the second coordinate and we define $R_{w_{n}}$ to be the $w_{n}$-radial section in $\mathbb{D}^{2}$ given by $\left.R_{w_{n}}=\left\{\left(r w_{n}, \zeta\right): 0 \leq r<1, \zeta \in \mathbb{D}\right\}\right)$;

(3) $\operatorname{Lat}\left(u_{1}(T), u_{2}(T)\right)=\operatorname{Lat}(T)$.

Proof. Let $g$ be the conformal transformation of $\mathbb{D}$ onto the domain $G$ defined in Lemma 4.2 , and then write $u_{1}=g \circ g, T_{1}=u_{1}(T)$. Using similar arguments to the ones used in [13] and [14], one can show that $\sigma_{l e}\left(T_{1}\right)$ is dominating for $E$, and moreover, $\sigma_{l e}\left(T_{1}\right)$ contains $\partial G$ with the possible exception of a finite length arc on $\partial G \backslash \mathbb{T}$. Without loss of generality, we may assume that $\sigma_{l e}\left(T_{1}\right) \supseteq \partial G$. One can remark that since $T$ is a completely nonunitary contraction, then so is $T_{1}=$ $u_{1}(T)$ [17], p.114. The smallest weak-operator-topology (WOT)-closed subalgebra of $\mathcal{L}(\mathcal{H})$ that contains $T$ and $I_{\mathcal{H}}$ will be denoted by $\mathcal{W}_{T}$. We also have that $\mathcal{W}_{T_{1}}=$ $\mathcal{W}_{T}$ and then $\operatorname{Lat}(T)=\operatorname{Lat}\left(T_{1}\right) ;$ cf., [13]. Note that

$$
\bigcup_{n \in \mathbb{N}} \lambda_{n}^{0} \subseteq \sigma_{l e}\left(T_{1}\right) \cap \mathbb{D}
$$


and since the function $h$ constructed in Lemma 4.1 is continuous on each $\lambda_{n}^{0}$, we obtain that

$$
\bigcup_{n \in \mathbb{N}} h\left(\lambda_{n}^{0}\right) \subseteq \sigma_{l e}\left(T_{2}\right) \cap \mathbb{D} .
$$

We then write $u_{2}=h \circ g \circ g$ and $T_{2}=u_{2}(T)$. By Corollary 3.2, we have that for $z \in \sigma_{l e}(T) \cap \mathbb{D}$

$$
\left(u_{1}(z), u_{2}(z)\right) \in \sigma_{l e}\left(T_{1}, T_{2}\right) .
$$

Therefore, by (4.3) and (4.4), we easily infer that

$$
\begin{array}{r}
{\left[\bigcup_{n \in \mathbb{N}} \lambda_{n}^{0}\right]^{-} \cap \mathbb{T} \supseteq E,} \\
{\left[\bigcup_{n \in \mathbb{N}} h\left(\lambda_{n}^{0}\right)\right]^{-} \cap \mathbb{T}=\mathbb{T},}
\end{array}
$$

and, moreover, by Lemma 4.2

$$
E \times \mathbb{T} \subseteq \sigma_{l e}\left(T_{1}, T_{2}\right) \cap \mathbb{T}^{2} .
$$

One can remark that a little change in the definition of the Carathéodory domain $G$ yields $E \times \mathbb{T}=\sigma_{l e}\left(T_{1}, T_{2}\right) \cap \mathbb{T}^{2}$.

For the dense subset of $E$ given by the sequence $\left\{w_{n}\right\}$ such that $\mathcal{C}_{\lambda_{n}^{0}}\left(h, w_{n}\right) \supseteq \mathbb{T}$, we may assume that $\lambda_{n}^{0} \subseteq \sigma_{l e}\left(T_{1}\right)$ (cf. [13]) and then (2) follows. (All we have to do is to be careful in choosing $\eta=\eta_{m, n}$ in the proof of Lemma 4.2, i.e., to be "small" also with respect to the distance from $\mathbb{T}$ to $h\left[\left(r_{m}-\delta_{m, n}\right) e^{i \theta_{n}}, r_{m} e^{i \theta_{n}}\right]$, and we get the desired nontangential convergence.) Since $E$ is a proper arc of $\mathbb{T}$, we have $\operatorname{Lat}(T)=\operatorname{Lat}\left(T_{1}\right) \subseteq \operatorname{Lat}\left(T_{2}\right)$, and (3) follows.

Corollary 4.4. If every pair of commuting completely nonunitary contractions satisfying conditions (1) and (2) in Theorem 4.3 has a nontrivial common invariant subspace, then every contraction of spectral radius one has a nontrivial invariant subspace.

Remark 4.5. The following consequences of Theorem 4.3 are also true:

(1) We remark that, in particular, with the same notation as above, $\sigma_{l e}\left(T_{2}\right)$ is dominating on $\mathbb{T}$ and then $T_{2}$ is a (BCP)-operator.

(2) For a pair $\left(S_{1}, S_{2}\right)$ of contractions in $\mathcal{L}(\mathcal{H})_{\text {comm }}^{(2)}$, we say that $\left(S_{1}, S_{2}\right) \in C_{0}^{(2)}$ if there exists a function $m$ in $\mathbf{H}^{\infty}\left(\mathbb{D}^{2}\right)$ such that $m\left(S_{1}, S_{2}\right)=0$. An example of a $C_{0}^{(2)}$-pair that has a large joint spectrum is presented in [18] (i.e., it is shown that $\left.\mathbb{T}^{2} \subseteq \sigma_{H}\left(S_{1}, S_{2}\right)\right)$. The construction is based upon a conformal mapping of a completely nonunitary contraction $T$ with an already large spectrum $(\sigma(T)=\mathbb{D})$. A little change in the preceding constructions shows that all we need is, in fact, for $T$ to be a contraction with spectral radius one. For instance, with the same notation as above, let us write $\tilde{T}_{1}=T_{1}^{2}$ and $\tilde{T}_{2}=$ $h\left(T_{1}^{2}\right)$. We remark that for the new pair $\left(\tilde{T}_{1}, \tilde{T}_{2}\right)$ in $\mathcal{L}(\mathcal{H})_{\text {comm }}^{(2)}(1)$ and $(2)$ in Theorem 4.3 are satisfied for $E=\mathbb{T}$. We also have that $\left(\tilde{T}_{1}, \tilde{T}_{2}\right) \in C_{0}^{(2)}$. However, the pair $\left(\tilde{T}_{1}, \tilde{T}_{2}\right)$ will no longer satisfy $(3)$ in Theorem 4.3 .

The next results are concerned with the existence of hyperinvariant subspaces for operators in $\mathcal{L}(\mathcal{H})$ and $\mathcal{L}(\mathcal{H})_{\text {comm }}^{(2)}$. 
Proposition 4.6. There exist functions $\hat{u}_{1}$ and $\hat{u}_{2}$ of norm one in $\mathbf{H}^{\infty}(\mathbb{D})$ such that for every completely nonunitary contraction $T$ with $1 \in \sigma(T)$,

$$
\sigma_{H}\left(\hat{u}_{1}(T), \hat{u}_{2}(T)\right)=\mathbb{D}^{2^{-}} .
$$

Proof. In the proof of Theorem 4.3 we have obtained two bounded analytic functions $u_{1}, u_{2}$ verifying in particular that $\sigma_{H}\left(u_{1}(T), u_{2}(T)\right) \supseteq E \times \mathbb{T}$. Let $C_{1}$ be a Cantor set in $E$ and $C_{2}$ in $\mathbb{T}$, and for each $j=1,2$, let $g_{j}$ be continuous maps of $C_{j}$ onto $\mathbb{D}^{-}$. Then, by the Carleson-Rudin theorem [15], p.58, there exists a function $f_{j}$ in $A(\mathbb{D})$ such that $\left.f_{j}\right|_{C}=g_{j}$ and $\left\|f_{j}\right\|_{\infty}=\left\|g_{j}\right\|_{\infty}=1$. Thus, using Theorem 3.5 for $T_{1}=u_{1}(T), T_{2}=u_{2}(T)$, and $\left(w_{1}, w_{2}\right) \in C_{1} \times C_{2}$, we infer that

$$
\left(f_{1}\left(w_{1}\right), f_{2}\left(w_{2}\right)\right) \in \sigma_{H}\left(f_{1}\left(T_{1}\right), f_{2}\left(T_{2}\right)\right)
$$

and therefore, by the projection property of the Harte spectrum

$$
\mathbb{D}^{2-} \subseteq f_{1}\left(C_{1}\right) \times f_{2}\left(C_{2}\right) \subseteq \sigma_{H}\left(f_{1}\left(T_{1}\right), f_{2}\left(T_{2}\right)\right) \subseteq \mathbb{D}^{2-} .
$$

For $j=1,2$, we define $\hat{u}_{j}=f_{j} \circ u_{j}$ and the result follows.

Corollary 4.7. If every pair of commuting completely nonunitary contractions for which the joint Harte spectrum contains the closed bidisc has a nontrivial hyperinvariant subspace, then every nonscalar contraction in $\mathcal{L}(\mathcal{H})$ with spectral radius one has a nontrivial hyperinvariant subspace.

Corollary 4.8. Let $E_{1}$ and $E_{2}$ be any two arcs of $\mathbb{T}$ that do not reduce to a point. If every pair of commuting completely nonunitary contractions for which the joint Harte spectrum contains the closed bidisc has a nontrivial hyperinvariant subspace, then every pair of commuting completely nonunitary contractions for which the joint Harte spectrum contains $E_{1} \times E_{2}$ has a nontrivial hyperinvariant subspace.

Proof. Let $\left(T_{1}, T_{2}\right)$ be a pair of completely nonunitary contractions in $\mathcal{L}(\mathcal{H})_{\text {comm }}^{(2)}$ such that $\sigma_{H}\left(T_{1}, T_{2}\right) \supseteq E_{1} \times E_{2}$. Then for $j=1,2$, we choose Cantor sets $C_{j} \subset E_{j}$ and define a continuous map $g_{j}$ from $C_{j}$ onto $\mathbb{D}^{-}$. Let $f_{j} \in A(\mathbb{D})$ be such that $\left.f_{j}\right|_{C_{j}}=g_{j}$. Applying Proposition 4.6, we infer that

$$
\sigma_{H}\left(f_{1}\left(T_{1}\right), f_{2}\left(T_{2}\right)\right)=\mathbb{D}^{2-},
$$

and, moreover,

$$
\operatorname{HLat}\left(f_{1}\left(T_{1}\right), f_{2}\left(T_{2}\right)\right) \subseteq \operatorname{HLat}\left(T_{1}, T_{2}\right)
$$

Remark. This paper is part of the author's Ph.D. thesis, written at Texas A\&M University under the direction of Professor Carl Pearcy. The author wishes to express his appreciation to Professor Theodore Gamelin for valuable information concerning this paper.

\section{REFERENCES}

[1] E. Albrecht and B. Chevreau. Invariant subspaces for certain representations of $\mathbf{H}^{\infty}(G)$, Functional Analysis, Biersted, Pietsch, Ruess, Vogt, Marcel Dekker, Inc., New York, 1994, p.293-305. MR 94i:47009

[2] O. B. Bekken. Rational approximation on product sets, Trans. Amer. Math. Soc., 191(1974), 301-316. MR 52:804

[3] E. Briem, A. M. Davie and B. K. Øksendal. Functional calculus for commuting contractions, J. London Math., 7(1974), 709-718 MR 48:9430 
[4] S. Brown, B. Chevreau and C. Pearcy. Contractions with rich spectrum have invariant subspaces, J. Operator Theory, 1(1979), 123-136. MR 80m:47002

[5] S. Brown, B. Chevreau and C. Pearcy. On the structure of contraction operators II, J. Funct. Anal., 76(1988), 30-55. MR 90b:47030b

[6] H. Bercovici and W. S. Li. Isometric functional calculus on the bidisk, Bull. London Math. Soc, 25(1993), 582-590. MR 94i:47010

[7] L. Brown, A. Shields and K. Zeller. On absolutely convergent exponential sums, Trans. Amer. Math. Soc., 96(1960), 162-183. MR 26:332

[8] C. Carathéodory. Über die Begrenzung einfachzusammenhängender Gebiete, Math. Ann., 73(1913), 323-370

[9] E. F. Collingwood and A. J. Lohwater. The theory of cluster sets, Cambridge University Press, London, 1966. MR 38:325

[10] R. E. Curto. Applications of several complex variables to multiparameter spectral theory, Surveys of recent results in Operator Theory I, Longman, London, 1988, p.25-90. MR 90d:47007

[11] R. E. Curto and L. A. Fialkow. Elementary operators with $\mathbf{H}^{\infty}$-symbols, Integral Equations and Operator Theory, 10(1987), 707-720. MR 88j:47016

[12] C. Foias and W. Mlak. The extended spectrum of completely nonunitary contractions and the spectral mapping theorem, Studia Math., 26(1966), 239-245. MR 34:610

[13] C. Foiaş and C. Pearcy. (BCP)-operators and enrichment of invariant subspace lattices, J. Operator Theory, 9(1983), 187-202. MR 85d:47005

[14] C. Foiaş, C. Pearcy and B. Sz.-Nagy Contractions with spectral radius one and invariant subspaces, Acta Sci. Math. (Szeged) 43(1981), 273-280. MR 84g:47002

[15] T. W. Gamelin. Uniform algebras, Chelsea, New York, 1984.

[16] M. Kosiek, A. Octavio and M. Ptak. On the reflexivity of pairs of contractions, Proc. Amer. Math. Soc., 123(1995), 1229-1236. MR 95e:47061

[17] B. Sz.-Nagy and C. Foiaş. Harmonic analysis of operators on Hilbert space, North Holland Publishing Co., Amsterdam-Budapest, 1970. MR 43:947

[18] A. Octavio. On the joint spectrum and $\mathbf{H}^{\infty}$-functional calculus for pairs of commuting contractions, Proc. Amer. Math. Soc., 114(1992), 495-503. MR 92j:47019

[19] W. Rudin. Function theory in the unit ball of $\mathbb{C}^{n}$, Springer Verlag, New York, 1980. MR 82i: 32002

[20] K. Rudol. Spectral mapping theorems for analytic functional calculi, Operator Theory: Advances and applications, Birkhhäuser Verlag, Basel, 1986, p.331-340. MR 88j:47017

[21] D. Sarason. Weak star generators of $\mathbf{H}^{\infty}$, Pacific J. Math., 17(1966), 519-528. MR 35:2151

Department of Mathematics and Computer Science, Texas Lutheran University, SEguin, TeXas 78155

Institute of Mathematics of the Romanian Academy, PO Box 1-764, RO-70700 Bucharest, Romania

Current address: Department of Mathematics and Computer Science, Texas Lutheran University, Seguin, Texas 78155

E-mail address: IONESCU_A@txlutheran.edu 